(C) 2020. This manuscript version is made available under the CC-BY-NC-ND 4.0 license http:// creativecommons.org/licenses/by-nc-nd/4.0/

RUNNING HEAD: Bullying, stigma, and mental health in adolescents living with HIV

\title{
Prospective associations between bullying victimisation, internalised stigma, and mental health in South African adolescents living with HIV
}

Mark E. Boyes ${ }^{1 *}$, Marija Pantelic ${ }^{2,3}$, Marisa Casale ${ }^{2,4}$, Elona Toska ${ }^{2,5,6}$, Elizabeth Newnham ${ }^{1,7}$, \& Lucie D. Cluver ${ }^{2,8}$

1: School of Psychology, Faculty of Health Sciences, Curtin University, Perth, Australia

2: Department of Social Policy and Intervention, University of Oxford, Oxford, UK

3: Brighton and Sussex Medical School, University of Sussex, Brighton, UK

4: School of Public Health, University of the Western Cape, Cape Town, South Africa

5: Centre for Social Science Research, University of Cape Town, Cape Town, South Africa

6: Department of Sociology, University of Cape Town, Cape Town, South Africa

7: FXB Center for Health and Human Rights, Harvard T.H. Chan School of Public Health, Boston, United States

8: Department of Psychiatry and Mental Health, University of Cape Town, Cape Town, South Africa

\section{Citation:}

Boyes, M. E., Pantelic, M., Casale, M., Toska, E., Newnham, E., \& Cluver, L. (2020). Prospective associations between bullying victimisation, internalised stigma, and mental health in South African adolescents living with HIV. Journal of Affective Disorders, 276, 418-423. DOI: 10.1016/j.jad.2020.07.101.

NOTICE: this is the author's version of a work that was accepted for publication in Journal of Affective Disorders. Changes resulting from the publishing process, such as peer review, editing, corrections, structural formatting, and other quality control mechanisms may not be reflected in this document. Changes may have been made to this work since it was submitted for publication. A definitive version was subsequently published in Journal of Affective Disorders [Volume 276, 2020] DOI: 0.1016/j.jad.2020.07.101.

\section{*Corresponding author:}

Dr Mark Boyes

Senior Research Fellow

NHMRC Emerging Leadership Fellow

School of Psychology, Faculty of Health Sciences

Curtin University, GPO Box U1987

Perth, Western Australia, 6845

Phone: +61892667025

Fax: +61 892662464

Email: mark.boyes@curtin.edu.au

Twitter:@me_boyo

Web: www.markboyes.com 
Abstract

Background: Adolescents living with HIV may be at elevated risk of psychological problems, which are correlated with negative health outcomes. In cross-sectional research with HIV-affected adolescents, bullying victimisation and internalised HIV stigma have been associated with poorer psychological health. We extended these findings and tested longitudinal associations between bullying victimisation, internalised stigma, and mental health among adolescents living with HIV. We also tested whether relationships between bullying victimisation and psychological symptoms were mediated by internalised stigma. Method: Adolescents living with HIV ( $n=1060,10-19$ years, 55\% female), who had ever initiated HIV treatment in 53 public health facilities in the Eastern Cape, South Africa, were interviewed and followed up 18 months later $(n=995,94 \%$ retention). Participants completed well-validated measures of depression, anxiety, posttraumatic stress, bullying victimisation, and internalised stigma. Results: After adjusting for baseline mental health and sociodemographic characteristics, baseline internalised stigma prospectively predicted poorer outcomes on all psychological measures. Bullying victimisation at baseline was not directly associated with any psychological measures at follow up; however, it was indirectly associated with all psychological measures via internalised stigma. Limitations: Reliance on self-report measures and poor reliability of the depression scale. Conclusions: Bullying victimisation is associated with internalised stigma, which in turn predicts psychological symptoms over time. Interventions reducing internalised stigma and associated psychological distress are needed, and these should be integrated into HIV care to ensure optimal HIV management. The implementation of bullying prevention programs may reduce internalised stigma and promote mental health among adolescents living with HIV.

Keywords: HIV, mental health, stigma, bullying victimisation, adolescents 
Bullying, stigma, and mental health in adolescents living with HIV 3

\section{Highlights}

- Among adolescents living with HIV, bullying is associated with internalised stigma

- Internalised stigma in turn predicts psychological symptoms over time

- Interventions reducing internalised stigma and psychological distress are needed

- Anti-bullying programs may reduce stigma among adolescents living with HIV

- Anti-bullying programs may also promote mental health among adolescents with HIV 
Bullying, stigma, and mental health in adolescents living with HIV 4

Introduction

Since 1950 the number of adolescents worldwide has more than doubled and in sub-Saharan Africa the number of adolescents is expected to double again by 2050. Additionally, although declining in all other age groups AIDS-related deaths among adolescents in sub-Saharan Africa are increasing. Adolescents therefore represent an increasing proportion of people living with HIV and are a key population in responding to HIV (UNICEF, 2018). Importantly, among both adults and adolescents affected by HIV, mental health difficulties are associated with negative health-related outcomes including risk of HIV transmission, morbidity and mortality due to non-adherence to antiretroviral therapy, as well as poor engagement in care (Mutumba et al., 2015; Remien et al., 2019). Therefore, addressing mental health difficulties among adolescents living with HIV, particularly in low-resource settings such as sub-Saharan Africa, is an emerging priority (Lowenthal et al., 2014). However, there are few well-established evidence-based programmes for adolescent mental health promotion in low and middle-income countries (Skeen et al., 2017). For effective programming, it is essential to identify risk and protective factors associated with psychological outcomes that can be targeted through interventions. In cross-sectional studies in the context of HIV, bullying victimisation (Boyes et al., 2014; Boyes et al., 2019) and internalised HIV stigma (Boyes et al., 2019; Pantelic et al., 2017) have been strongly linked with poorer mental health and are the focus of the current research. Importantly, there is evidence that both bullying victimisation (Ttofi and Farrington, 2011; Vreeman and Carroll, 2007) and internalised stigma (Pantelic et al., 2019) are amenable to intervention.

Bullying refers to repeated acts of aggressive behaviour intended to cause harm, and is typically characterised by an imbalance in power between the perpetrator and the victim (Rigby, 2002). Bullying victimization in South Africa is widespread, with data from a 
nationally representative sample of high school students putting prevalence rates at $41 \%$ (Reddy et al., 2003). Experiences of bullying victimization peak in early adolescence, a period during which children increasingly spend time with their peers and place more value on peer relationships and approval. The accumulation of evidence from both high-income as well as low and middle-income countries offers strong support for a relationship between bullying victimization and poor mental health outcomes (Arseneault et al., 2010; Boyes et al., 2014; Fekkes et al., 2006). Specifically, in general populations bullying victimisation is robustly associated with poorer psychosocial outcomes, in particular internalising symptoms such as depression and anxiety, even after adjusting for the effects of initial internalising symptoms. Additionally, longitudinal research, showing dose response associations between bullying victimisation and internalising symptoms (across different samples and using differing measures), offers further support for links between bullying victimisation and psychological outcomes (Arseneault et al., 2010).

Adolescents affected by HIV are often perceived more negatively by their peers (Ishikawa et al., 2011) and members of an "out group" are more likely to be victims of bullying (Burns et al., 2008; Gini, 2006). Given this, it is perhaps unsurprising that there is emerging evidence of a link between bullying victimisation and mental health in the context of HIV. Caregiver HIV/AIDS sickness is a risk factor for bullying victimisation (Cluver et al., 2010) and bullying victimisation has also been demonstrated to mediate associations between familial HIV and symptoms of depression and anxiety in South African adolescents (Boyes and Cluver, 2015). More specifically, for adolescents living with HIV, being bullied for taking medication has been associated with depression in Malawi (Kim et al., 2015) and bullying victimisation was found to be associated with worse outcomes on all mental health measures (depression, anxiety, posttraumatic stress, and conduct problems) administered in a recent study of over a thousand South African adolescents living with HIV (the baseline data 
for the current study, Boyes et al., 2019). Additionally, bullying victimisation has been associated with increased HIV/AIDS related stigma among HIV affected South African adolescents (Cluver et al., 2010).

HIV stigma refers to negative attitudes and prejudice directed at people living with HIV (UNAIDS, 2015). HIV stigma is strongly associated with poor mental health outcomes, particularly when this stigma is internalised (Pantelic et al., 2015; Rueda et al., 2016). Internalisation of HIV stigma occurs when individuals living with HIV accept perceived negative public attitudes towards people living with HIV as applicable to themselves (Pantelic et al., 2015). By evoking strong feelings of shame and worthlessness, internalised stigma is consistently linked to negative psychological and health-related outcomes among people living with HIV (Pantelic et al., 2015; Pantelic et al., 2019; Rueda et al., 2016). Although most research on links between internalised stigma and mental health is conducted in adult samples, in our recent study of South African adolescents living with HIV we observed cross-sectional associations between internalised stigma and symptoms of depression, anxiety, and posttraumatic stress (Boyes et al., 2019). Additionally, potentially linking experiences of bullying and internalised stigma, victimisation and violence exposure are argued to be key drivers of internalised HIV stigma (Abadía-Barrero and Castro, 2006; Pantelic et al., 2017). Consistent with this view, bullying victimisation and HIV stigma are correlated, at least cross-sectionally, among adolescents living with and affected by HIV (Boyes and Cluver, 2015; Pantelic et al., 2017).

While there is an emerging body of literature establishing associations between bullying victimisation, internalised stigma, and adolescent mental health in the context of HIV, this literature is almost exclusively cross-sectional, therefore precluding any conclusions regarding the direction of these associations. We aimed to extend the current literature and test longitudinal associations between bullying victimisation, internalised 
stigma, and mental health among adolescents living with HIV. It was hypothesised that both bullying victimisation and internalised stigma would be prospectively associated with symptoms of depression, anxiety, and posttraumatic stress. Additionally, as victimisation is argued to be a driver of internalised stigma over time, we also tested if relationships between bullying victimisation and psychological symptoms were mediated by internalised stigma.

Method

\section{Participants}

As reported previously (Boyes et al., 2019; Cluver et al., 2018b), between March 2014 and September 2015 adolescents living with HIV $(n=1060,10-19$ years, 55\% female) were recruited from a health district in the Eastern Cape province, South Africa. All healthcare facilities providing antiretroviral therapy were visited $(n=83)$ and all facilities with more than five antiretroviral therapy eligible adolescents were included in the study $(n=39)$. For each facility, all adolescents who had initiated antiretroviral therapy were identified $(n=1176)$. Addresses were recorded from patient files for community-tracing. Over the course of the study, the Department of Health implemented a down-referral program, during which adolescents in the initial facilities were transferred to 53 healthcare facilities. Adolescents were recruited at clinics where they were receiving antiretroviral therapy, or traced into their communities. In total, $90.1 \%$ of all study-eligible adolescents were interviewed at baseline. The majority of those who did not participate did not provide adolescent and/or caregiver consent (4.1\%) or were untraceable (3.9\%). Adolescents were reinterviewed on average 18 months later in a second wave of data collection, with $94 \%$ retention (Haghighat et al., 2019). 


\section{Measures}

Mental health was measured at both baseline and follow up assessment. All scales are validated and have been used previously with South African adolescents living in high HIVprevalence communities (Cluver et al., 2013).

Depression: Symptoms of depression (past two weeks) were measured with eight items (Baseline: $\omega=0.55$; Follow up: $\omega=0.46$ ) from the widely-used and well-validated Child Depression Inventory - Short Form (Kovacs, 1992). Each symptom is presented in three phrases, and respondents are asked to select the option that best represents their feelings (e.g. 0: "I am sad once in a while", 1: "I am sad many times", 2:"I am sad all the time"). Items are summed to generate a total score, with higher scores indicative of more depressive symptomatology.

Anxiety: Symptoms of anxiety were measured using an abbreviated version of the Revised Children's Manifest Anxiety Scale (Reynolds and Richmond, 1978). The full scale has been validated in South Africa (Boyes and Cluver, 2013). The 14 highest loading items (Baseline: $\omega=0.80$; Follow up: $\alpha=0.84$ ) identified previously through factor analysis (Cluver et al., 2013) were administered at both time points. Items (e.g. "I worry a lot of the time") are responded to on a binary scale ( $0:$ No; $1:$ Yes) and are summed to give a total score, with higher scores indicating more anxiety.

Posttraumatic stress: Symptoms of posttraumatic stress (past month) were measured with an abbreviated version of the Child PTSD Checklist (Amaya-Jackson et al., 1995). The full checklist has been validated in South Africa (Boyes et al., 2012). The 19 highest loading items (Baseline: $\omega=0.90$; Follow up: $\omega=0.92$ ) identified in a previous study (Cluver et al., 2013) were administered at both time points. Prior to completing the checklist children identify the most upsetting or frightening thing that has happened to them. Items (e.g. "When something reminds you of what happened, do you get tense or upset?") are responded to on a 
four-point frequency scale $(0:$ Not at all; 1 : Some of the time; 2: Most of the time; $3:$ All the time). Items are summed to give a total score, with higher scores indicative of more posttraumatic stress symptomatology.

Bullying victimisation: Bullying victimisation was measured using the nine-item $(\omega=0.79)$ Social and Health Assessment Peer Victimisation Scale (Ruchkin et al., 2004). Items are rated according to frequency in the past year (1: Never, 2: Once, 3: Two to three times 4: Four or more times) and the scale has been used previously with this age group in South Africa (Boyes and Cluver, 2015; Boyes et al., 2014; Cluver et al., 2010). The scale assesses physical (e.g. "punched, kicked, or beat me up”), verbal (e.g. "called me names or swore at me"), and relational (e.g. "refused to talk to me or made other people not talk to me") bullying victimisation. One item assesses invasion of physical space. Total bullying victimisation scores were calculated by summing all items, with higher scores indicative of more bullying victimisation.

Internalised HIV stigma: Internalised stigma (past year) was measured at baseline assessment using the five-item $(\omega=0.75)$ internalised stigma subscale from the Adolescents Living with HIV Stigma Scale (Pantelic et al., 2018). Items are presented alongside a gender specific vignette [This is Lundi/Nosizi. Living with HIV is difficult for him sometimes. Some days Lundi feels ashamed and he struggles to feel good about himself. Could you say how much these things have been true for you in the past year?]. Items (e.g. "Sometimes Lundi feels ashamed that he is HIV-positive. Do you ever feel this way?") are responded to on a three-point likert scale ( $0:$ Not at all, 1: Sometimes, 2: All the time). Items were summed to generate a total score, with higher scores indicating more internalised stigma.

Sociodemographic information: Sociodemographic information included adolescent age, gender, urban versus rural location, and household poverty. Household poverty was measured using an index of access to the eight highest socially-perceived necessities (e.g. 
three meals per day) for South African children and adolescents (Barnes and Wright, 2012). These items were responded to on a binary scale ( 0 : Has access; 1 : Does not have access) and were summed to generate a total poverty score.

\section{Procedure}

Ethical approvals were obtained from the Universities of Oxford and Cape Town, Eastern Cape Departments of Health and Basic Education, and participating hospitals. In order to avoid unintentional disclosure and stigma, recruitment processes and documentation presented the study within communities and organisations as investigating health, education and support service access for adolescents who have had any extended contact with the health system and were on long-term treatment. Written informed consent was obtained from all primary caregivers and all adolescents provided written assent. Questionnaires were administered by trained research assistants or via tablet-assisted self-interviewing, depending on literacy level, and participation took approximately 90 minutes. No financial incentive for participation was provided but all participants received a certificate of participation, snacks, and a small gift pack including basic items like pencils.

\section{Analyses}

We analysed the data in four phases using SPSS 25 and Mplus 8.3. First, we checked for any differences in sociodemographic characteristics, bullying victimisation, internalised stigma, and depression, anxiety, and posttraumatic stress scores between participants lost and retained at follow up. Second, we calculated descriptive statistics for bullying victimisation, internalised stigma, and psychological symptoms. Third, correlations summarised bivariate relationships between bullying victimisation and internalised stigma (measured at baseline assessment) and psychological symptoms (measured at baseline and follow up assessments). 
Finally, we tested predicted prospective direct and indirect effects of bullying victimisation and internalised stigma on symptoms of depression, anxiety, and posttraumatic stress in a path model. With the exception of analyses comparing adolescents lost and retained at follow up, the analyses were limited to participants assessed at both data collection points.

\section{Results}

\section{Adolescents lost and retained at follow up}

Adolescents lost to follow up $\left(n=69,6.5 \% ; M_{a g e}=14.67, S D=3.03\right)$ were significantly older than those retained $\left(M_{\text {age }}=13.77, S D=2.83\right)$ in the study, $[F(1,1063)=6.39, p=$ 0.012] and were significantly more likely to live in an urban location at baseline assessment, $\chi^{2}(1)=5.05, p=0.025$. There were no differences in gender $\left[\chi^{2}(1)<0.001, p=0.994\right]$ baseline poverty $[F(1,1062)=0.52, p=0.472]$, bullying victimisation $[F(1,1063)=0.87, p$ $=0.351]$, internalised stigma $[F(1,1019)=1.28, p=0.259]$, depression $[F(1,1063)=2.22, p$ $=0.136]$, anxiety $[F(1,1063)=0.15, p=0.700]$, or posttraumatic stress $[F(1,1063)=2.29, p$ $=0.130]$ scores.

\section{Descriptive statistics and bivariate correlations}

Descriptive statistics, disaggregated by gender, as well as Pearson correlations between the variables of interest are summarised in Table 1. Baseline bullying victimisation scores were significantly correlated with baseline depression, anxiety, and posttraumatic stress scores, as well as depression and anxiety scores at follow up assessment. Baseline internalised stigma scores were significantly correlated with baseline bullying victimisation and all psychological outcomes at both baseline and follow up assessments. 


\section{(Insert Table 1 approximately here)}

Associations between bullying victimisation, internalised stigma, and symptoms of depression, anxiety, and posttraumatic stress

The hypothesised prospective associations between bullying victimisation and internalised stigma (measured at baseline assessment), and symptoms of depression, anxiety, and posttraumatic stress (measured on average 18 months later) were tested in a path model in Mplus 8.3 using maximum likelihood estimation ${ }^{1}$. We evaluated overall model fit using the chi-square statistic $\left(\chi^{2}\right)$, which should be non-significant. Additionally, we also report Comparative Fit Index (CFI), Tucker Lewis Index (TLI), Root Mean Square Error of Approximation (RMSEA), and Standardised Root Mean Square Residual (SRMR). For CFI and TLI, values of 0.95 or greater indicates good fit. For RMSEA and SRMR, values of 0.05 or less indicates good fit. Baseline depression, anxiety, and posttraumatic stress scores were controlled for in the analysis. Additionally, given significant associations between gender, age, poverty, urban versus rural location, and many of the variables under investigation, we also adjusted for these.

After adjusting for mental health, poverty, urban/rural location, age, and gender, bullying victimisation at baseline was significantly associated with baseline internalised stigma $(\beta=0.08, p=0.008)$. Baseline internalised stigma scores predicted higher scores on all measures of psychological symptoms at follow up assessment (Depression: $\beta=0.10, p=$ 0.002; Anxiety: $\beta=0.13, p<0.001$; Posttraumatic stress: $\beta=0.10, p=0.003$ ). Depression, anxiety, and posttraumatic stress scores were positively correlated (Figure 1). Bullying

\footnotetext{
${ }^{1}$ As many of the variables were not normally distributed, we also used a bootstrapping procedure to test the hypothesised associations between bullying victimisation, internalised stigma, and symptoms of depression, anxiety, and posttraumatic stress. Direct and indirect effects (and bias corrected $95 \%$ confidence intervals) were estimated using 5000 bootstrapped re-sampling draws. We assessed statistical significance for all effects by examining whether the bias corrected $95 \%$ confidence intervals overlapped with zero. The pattern of findings did not differ from those reported in text.
} 
victimisation at baseline assessment was not directly associated with any psychological outcomes at follow up (Depression: $\beta=0.05, p=0.165$, Anxiety: $\beta=0.01, p=0.780$, Posttraumatic stress: $\beta=-0.01, p=0.722)$; however, it was indirectly associated with symptoms of depression $(\beta=0.01, p=0.048)$, anxiety $(\beta=.01, p=.030)$, and posttraumatic stress $(\beta=0.02, p=0.049)$ via internalised stigma. The final model fit the data well $\left[\chi^{2}(16)=\right.$ $19.34, p=0.252, \mathrm{CFI}=0.996, \mathrm{TLI}=0.990, \mathrm{RMSEA}=0.015(90 \% \mathrm{CI}=<0.001-0.035)$, SRMR $=0.022]$ and accounted for $6 \%$ of the variance in anxiety scores and $7 \%$ of the variance in depression and posttraumatic stress scores.

\section{(Insert Figure 1 approximately here)}

\section{Discussion}

We tested prospective associations between bullying victimisation, internalised stigma, and mental health in a large sample of South African adolescents living with HIV. We also tested if relationships between bullying victimisation and psychological symptoms were mediated by internalised stigma. Bullying victimisation and internalised stigma were salient predictors of increases in psychological symptoms over time among adolescents living with HIV. Specifically, while bullying victimisation did not exert direct effects on any of the psychological outcomes assessed, it was associated with internalised stigma, which in turn prospectively predicted symptoms of depression, anxiety, and posttraumatic stress. These findings extend previous cross-sectional research linking bullying victimisation (Boyes et al., 2019; Kim et al., 2015), internalised stigma (Boyes et al., 2019; Pantelic et al., 2017), and adolescent mental health in the context of HIV, and are consistent with suggestions that victimisation may be a risk factor for internalisation of HIV stigma (Abadía-Barrero and 
Castro, 2006; Pantelic et al., 2017). Taken together, current findings indicate that interventions to reduce internalised stigma and experiences of bullying victimisation may reduce psychological symptoms and promote mental health among adolescents living with HIV. Importantly, both internalised stigma (Pantelic et al., 2019) and bullying victimisation (Ttofi and Farrington, 2011; Vreeman and Carroll, 2007) are amenable to intervention.

A recent systematic review of interventions to reduce internalised HIV stigma reported that cognitive behavioural therapy was associated with reductions in internalised stigma (Pantelic et al., 2019). Additionally, cognitive behavioural therapy is an effective treatment for emotional disorders generally (Hofmann et al., 2012), and is also associated with improved medication adherence in the context of HIV (Safren et al., 2009). Cognitive behavioural therapy therefore appears a promising intervention to consider at the individual level, particularly if it can be integrated into HIV care to ensure optimal HIV management. However, the scalability of individualised interventions (such as cognitive behavioural therapy) in the context of strained health care systems in low and middle-income countries may be limited. Fortunately, there is also evidence that a number of structural interventions are effective in reducing internalised HIV stigma, including the provision of antiretroviral therapy, social empowerment, and economic strengthening (Pantelic et al., 2019). Improving these structural factors is closely aligned with the United Nations Sustainable Development Goals (United Nations, 2015). Monitoring internalised stigma and mental health among adolescents living with HIV in contexts where Sustainable Development Goals are being systematically addressed will provide valuable opportunities for evaluating the impacts of structural change on adolescent mental health, and also further theoretical understanding of the links between structural risks, internalised HIV stigma, and mental health.

Additionally, there is evidence from systematic reviews (Vreeman and Carroll, 2007) and meta-analyses (Ttofi and Farrington, 2011) for the effectiveness of anti-bullying 
interventions in high income countries, particularly when interventions incorporate a wholeschool approach involving multiple disciplines and the whole school community (Vreeman and Carroll, 2007). There is also evidence that reductions in bullying victimisation are associated with improvements in child mental health (Williford et al., 2012). Future research should trial bullying prevention programs in high HIV-prevalent areas. Targeting bullying victimization has two additional advantages. First, adopting a whole school approach reduces the risk of adolescents living with HIV being singled out and potentially exposed to more HIV stigma. Second, given associations between bullying victimization and adolescent mental health in South Africa more broadly (Boyes et al., 2014), bullying prevention efforts are likely to promote mental health for South African youth in general, while also benefitting vulnerable groups, such as adolescents living with HIV.

The study had a number of limitations that should be acknowledged. Although retention rates were exceptionally high given the context (94\%), we were not able to trace all adolescents and older adolescents disproportionately lost to follow up. In longitudinal research more vulnerable participants are generally at increased risk of being lost to follow up and our findings need to be considered with this in mind, as they may underestimate associations between bullying victimisation, internalised stigma, and psychological symptoms. There are also measurement considerations that should be noted. Specifically, the measure of bullying victimisation does not assess the reasons why individuals were targeted, and we therefore do not know if adolescents experienced victimisation because of their HIV status. The reliability of the depression measure was poor and local clinical cut-offs are not available for any of the mental health scales. Additionally, the reliance on self-report measures introduces risk of method overlap bias, as adolescents with mental health problems may feel more isolated and threatened and therefore perceive higher levels of victimization. Although the use of longitudinal data does reduce this issue to an extent, as we can control 
for baseline mental health scores, incorporating teacher or peer reports of bullying into future studies could be considered. However, this will likely be difficult when conducting research with adolescents living with HIV due to issues around confidentiality and stigma.

Additionally, the observed effects were small and the model only accounted for a small proportion of the variance in depression, anxiety, and posttraumatic stress measured at follow up assessment. Future research should investigate additional factors potentially associated with mental health over time among adolescents living with HIV. Importantly, if these factors are to be potential intervention targets, there should be evidence that they are amenable to change. For example, in previous cross-sectional research positive parenting was associated with lower emotional and behavioural difficulties among adolescents living with HIV (Boyes et al., 2019). There is also evidence from South Africa that parenting programs are effective in improving parenting behaviour in high poverty contexts (Cluver et al., 2018a). Additionally, social support has demonstrated protective effects on depression among adolescents living with HIV (Casale et al., 2019), and is another potentially malleable factor that warrants further investigation. Finally, it should be noted that the study was limited to South Africa and the findings may therefore not be representative of other low and middleincome countries.

Bearing these limitations in mind, our findings extend previous cross-sectional research and demonstrate that bullying victimisation is associated with internalised HIV stigma, which in turn predicts psychological symptoms over time. Given robust links between poor mental health and negative health-related outcomes in the context of HIV, interventions promoting mental health, reducing internalised stigma, and reducing bullying are needed. In particular, potentially scalable structural level interventions (e.g. provision of antiretroviral therapy, social empowerment, and economic strengthening) and universal school-based 
Bullying, stigma, and mental health in adolescents living with HIV 17

bullying prevention programs may be promising candidates for promoting mental health among adolescents living with HIV. 


\section{References}

Abadía-Barrero, C., Castro, A., 2006. Experiences of stigma and access to HAART in children and adolescents living with HIV/AIDS in Brazil. Social Science and Medicine 62, $1219-1228$

Amaya-Jackson, L., McCarthy, G., Cherney, M.S., Newman, E., 1995. Child PTSD Checklist $($. Duke University Medical Center, Durham, NC.

Arseneault, L., Bowes, L., Shakoor, S., 2010. Bullying victimization in youths and mental health problems: 'Much ado about nothing'? Psychological Medicine 40, 717-729.

Barnes, H., Wright, G., 2012. Defining child poverty in South Africa using the socially percieved necessities approach, In: Minujin, A. (Ed.), Global Child Poverty and Well-Being: Measurement, Concepts, Policy and Action. The Policy Press, Bristol.

Boyes, M.E., Cluver, L., 2013. Performance of the Revised Children's Manifest Anxiety Scale in a sample of children and adolescents from poor urban communities in Cape Town. European Journal of Psycholgical Assessment 29, 113-120.

Boyes, M.E., Cluver, L., 2015. Relationships between familial HIV/AIDS and symptoms of anxiety and depression: The mediating effect of bullying victimization in a prospective sample of South African children and addolescents. Journal of Youth and Adolescence 44, 847-859. 
Boyes, M.E., Cluver, L., Bowes, L., Ward, C., Badcock, N., 2014. Bullying victimisation, internalising symptoms, and conduct problems in South African children and adolescents: A longitudinal investigation. Journal of Abnormal Child Psychology 42, 1313-1324.

Boyes, M.E., Cluver, L., Gardner, F., 2012. Psychometric properties of the Child PTSD Checklist in a community sample of South African children and adolescents. PLOS ONE 7, e46905.

Boyes, M.E., Cluver, L.D., Meinck, F., Casale, M., Newnham, E., 2019. Mental health in South African adolescents living with HIV: correlates of internalising and externalising symptoms. AIDS Care 31, 95-104.

Burns, S., Maycock, B., Cross, D., Brown, G., 2008. The power of peers: Why some students bully others to conform. Qualitative Health Research 18, 1704-1716.

Casale, M., Boyes, M.E., Pantelic, M., Toska, E., Cluver, L.D., 2019. Suicidal thoughts and behaviour among South African adolescents living with HIV: Can social support buffer the impact of stigma? Journal of Affective Disorders 245, 82-90.

Cluver, L., Meinck, F., Steinert, J., Shenderovich, Y., Doubt, J., Herro Romero, R., Lombard, C., Redfern, A., Ward, C., Tsoanyane, S., Nzima, D., Sibanda, N., Wittesaele, C., DeStone, S., Boyes, M., Catanho, R., Lachman, J., Salah, N., Gardner, F., 2018a. Parenting for Lifelong Health: A pragmatic cluster randomised controlled trial of a non-commercialised parenting programme for adolescents and their families in South Africa. BMJ Global Health. 
Cluver, L.D., Bowes, L., Gardner, F., 2010. Risk and protective factors for bullying victimization among AIDS-affected and vulnerable children in South Africa Child Abuse and Neglect 34, 793-803.

Cluver, L.D., Orkin, M., Boyes, M.E., Sherr, L., Makasi, D., Nikelo, J., 2013. Pathways from parental AIDS to child psychological, educational, and sexual risk: Developing an empirically-based interactive theoretical model. Social Science and Medicine 87, 185-193.

Cluver, L.D., Pantelic, M., Toska, E., Orkin, M., Casale, M., Bungane, N., Sherr, L., $2018 b$. STACKing the odds for adolescent survival: health service factors associated with full retention in care and adherence amongst adolescents living with HIV in South Africa. Journal of the International AIDS Society 21, e25176.

Fekkes, M., Pijpers, F., Fredriks, A., Vogels, T., Verloove-Vanhorick, S., 2006. Do bullied children get ill, or do ill children get bullied? A prospective cohort study on the relationship between bullying and health-related symptoms. Pediatrics 117, 1568-1574.

Gini, G., 2006. Bullying as a social process: The role of group membership in students' perception of inter-group aggression at school. Journal of School Psychology 44, 51-65.

Haghighat, R., Toska, E., Cluver, L.D., Gulaid, L., Mark, D., Bains, A., 2019. Transition pathways out of pediatric care and associated HIV outcomes for adolescents living with HIV in South Africa. Journal of Acquired Immune Deficiency Syndromes 82, 166-174. 
Hofmann, S., Asnaani, A., Vonk, I., Sawyer, A., Fang, A., 2012. The efficacy of Cognitive Behavioral Therapy: A review of meta-analyses. Cognitive Therapy and Research 36, 427440.

Ishikawa, N., Pridmore, P., Carr-Hill, R., Chaimuangdee, K., 2011. The attitudes of primary schoolchildren in Northern Thailand towards their peers who are affected by HIV and AIDS. AIDS Care 23, 237-244.

Kim, M., Mazenga, A., Yu, X., Devandra, A., Nguyen, C., Ahmed, S., Kazembe, P., Sharp, C., 2015. Factors associated with depression among adolescents living with HIV in Malawi. BMC Psychiatry 15, 264.

Kovacs, M., 1992. Children's Depression Inventory. Multi-health Systems, Niagra Falls, NY.

Lowenthal, E., Bakeera-Kitaka, S., Marukuira, T., Chapman, J., Goldrath, K., Ferrand, R., 2014. Perinatally acquired HIV infection in adolescents from sib-Saharan Africa: A review of emerging challenges. The Lancet Infectious Diseases 14, 627-639.

Mutumba, M., Resnicow, K., Bauermeister, J., Harper, G., Musiime, V., Snow, R., Lepkowski, J., 2015. Development of a psychosocial distress measure for Ugandan adolescents living with HIV. AIDS and Behavior 19, 380-392.

Pantelic, M., Boyes, M.E., Cluver, L., Meinck, F., 2017. HIV, violence, blame and shame: Pathways of risk to internalized HIV stigma among South African adolescents living with HIV. Journal of the International AIDS Society 20, 21771. 
Pantelic, M., Boyes, M.E., Cluver, L.D., Thabeng, M., 2018. 'They say HIV is a punishment from God or from ancestors': Cross-cultural adaptation and psychometric assessment of an HIV stigma scale for South African adolescents living with HIV (ALHIV-SS). Child Indicators Research 11, 207-223.

Pantelic, M., Shenderovich, Y., Cluver, L.D., Boyes, M.E., 2015. Predictors of internalised HIV-related stigma: A systematic review of studies in sub-Saharan Africa. Health Psychology Review 9, 469-490.

Pantelic, M., Steinert, J., Park, J., Mellors, S., Murau, F., 2019. 'Management of a spoiled identity': Systematic review of interventions to address self-stigma among people living with and affected by HIV. BMJ Global Health 4, e001285.

Reddy, S., Panday, S., Swart, D., Jinabhai, C., Amosun, S., 2003. Umthenthe Uhlaba Usamila—The South African youth risk behaviour survey 2002. South African Medical Research Council, Cape Town.

Remien, R., Stirratt, M., Nguyen, N., Robbins, R., Pala, A., Mellins, C., 2019. Mental health and HIV/AIDS: The need for an integrated response. AIDS 33, 1411-1420.

Reynolds, C., Richmond, B., 1978. What I think and feel: A revised measure of children's anxiety. Journal of Abnormal Child Psychology 6, 271-280.

Rigby, K., 2002. New perspectives on bullying. Jessica Kingsley, London. 
Ruchkin, V., Schwab-Stone, M., Vermeiren, R., 2004. Social and Health Assessment (SAHA) psychometric development summary. Yale University, New Haven.

Rueda, S., Mitra, S., Chen, S., Gogolishvili, D., Globerman, J., Chambers, L., Wilson, M., Logie, C., Shi, Q., Morassaei, S., Rourke, S., 2016. Examining the associations between HIVrelated stigma and health outcomes in people living with HIV/AIDS: a series of metaanalyses. BMJ Open 6, e011453.

Safren, S., O'Cleirigh, C., Tan, J., Raminani, S., Reilly, L., Otto, M., Mayer, K., 2009. A randomized controlled trial of Cognitive Behavioral Therapy for Adherence and Depression (CBT-AD) in HIV-infected individuals. Health Psychology 28, 1-10.

Skeen, S., Sherr, L., Croome, N., Ghandi, N., Roberts, K., Macedo, A., Tomlinson, M., , 2017. Interventions to improve psychosocial well-being for children affected by HIV and AIDS: A systematic review. Vulnerable Children and Youth Studies 12, 91-116.

Ttofi, M., Farrington, D.P., 2011. Effectiveness of school-based programs to reduce bullying: A systematic and meta-analytic review. Journal of Experimental Criminology 7, 27-56.

UNAIDS, 2015. On the fast-track to end AIDS by 2030: Focus on location and population. UNAIDS, Geneva.

UNICEF, 2018. Turning the tide against AIDS will require more concentrated focus on adolescents and young people, https://data.unicef.org/topic/hivaids/adolescents-youngpeople/\# 
Bullying, stigma, and mental health in adolescents living with HIV 24

United Nations, 2015. Transforming our world: The 2030 agenda for sustainable development A/RES/70/1. United Nations.

Vreeman, R., Carroll, A., 2007. A systematic review of school-based interventions to prevent bullying. Archives of Pediatric and Adolescent Medicine 161, 78-88.

Williford, A., Boulton, A., Noland, B., Little, T.D., Karna, A., Salmivalli, C., 2012. Effects of the KiVa anti-bullying program on adolescent's depression, anxiety, and perception of peers. Journal of Abnormal Child Psychology 40, 289-300. 
Table 1. Descriptive statistics and correlations between bullying victimisation, internalised stigma, and mental health measures.

\begin{tabular}{|c|c|c|c|c|c|c|c|c|c|c|}
\hline & $\begin{array}{l}\text { Male } \\
(n=447) \\
M(S D)\end{array}$ & $\begin{array}{l}\text { Female } \\
(n=549) \\
M(S D)\end{array}$ & $\begin{array}{l}\text { Bullying } \\
\text { Victimisation }\end{array}$ & $\begin{array}{l}\text { Internalised } \\
\text { Stigma }\end{array}$ & $\begin{array}{l}\text { Depression } \\
\text { T1 }\end{array}$ & $\begin{array}{l}\text { Anxiety } \\
\text { T1 }\end{array}$ & $\begin{array}{l}\text { PTS } \\
\text { T1 }\end{array}$ & $\begin{array}{l}\text { Depression } \\
\text { T2 }\end{array}$ & $\begin{array}{l}\text { Anxiety } \\
\text { T2 }\end{array}$ & $\begin{array}{l}\text { PTS } \\
\text { T2 }\end{array}$ \\
\hline \multicolumn{11}{|l|}{ Baseline } \\
\hline $\begin{array}{l}\text { Bullying } \\
\text { victimisation }\end{array}$ & $11.20(2.93)$ & $11.44(3.49)$ & -- & $0.24 * * *$ & $0.22 * * *$ & $0.38 * * *$ & $0.27 * * *$ & $0.11^{* * *}$ & $0.10^{* *}$ & 0.05 \\
\hline $\begin{array}{l}\text { Internalised } \\
\text { stigma }\end{array}$ & $0.45(1.07)$ & $0.56(1.23)$ & & -- & $0.34 * * *$ & $0.42 * * *$ & $0.36^{* * *}$ & $0.17 * * *$ & $0.19 * * *$ & $0.18^{* * *}$ \\
\hline Depression $\mathrm{T} 1$ & $1.12(1.75)$ & $1.34(2.14)$ & & & -- & $0.42 * * *$ & $0.31 * * *$ & $0.23 * * *$ & $0.16^{* * *}$ & $0.15 * * *$ \\
\hline Anxiety T1 & $1.93(2.34)$ & $2.38(2.88)^{* *}$ & & & & -- & $0.53^{* * *}$ & $0.14 * * *$ & $0.21 * * *$ & $0.12 * * *$ \\
\hline $\begin{array}{l}\text { Posttraumatic } \\
\text { stress T1 }\end{array}$ & $4.24(5.82)$ & $5.65(7.33)^{* *}$ & & & & & -- & $0.16^{* * *}$ & $0.17 * * *$ & $0.21 * * *$ \\
\hline Follow up & & & & & & & & & & \\
\hline Depression T2 & $0.70(1.34)$ & $0.86(1.55)$ & & & & & & -- & $0.46^{* * *}$ & $0.27 * * *$ \\
\hline
\end{tabular}


Bullying, stigma, and mental health in adolescents living with HIV 26

$\begin{array}{lll}\text { Anxiety T2 } & 0.69(1.74) & 1.04(2.03)^{* *} \\ \text { Posttraumatic } & 2.83(5.11) & 3.64(6.31)^{*}\end{array}$

stress T2

Note: $\mathrm{T} 1=$ baseline assessment, $\mathrm{T} 2=18$ month follow up assessment. Gender differences are tested with one-way ANOVAs. ${ }^{*} p<0.05 * * p<$ $0.01 * * * p<0.001 . \mathrm{PTS}=$ posttraumatic stress. 
Figure 1. Associations between bullying victimisation, internalised stigma, and symptoms of depression, anxiety, and posttraumatic stress (Note: Bullying victimisation and internalised stigma measured at baseline assessment. Depression, anxiety, and posttraumatic stress scores measured at follow up assessment. $* * * p<0.001 * * p<0.01$. Solid lines $=$ significant associations. Dashed lines $=$ non-significant associations).

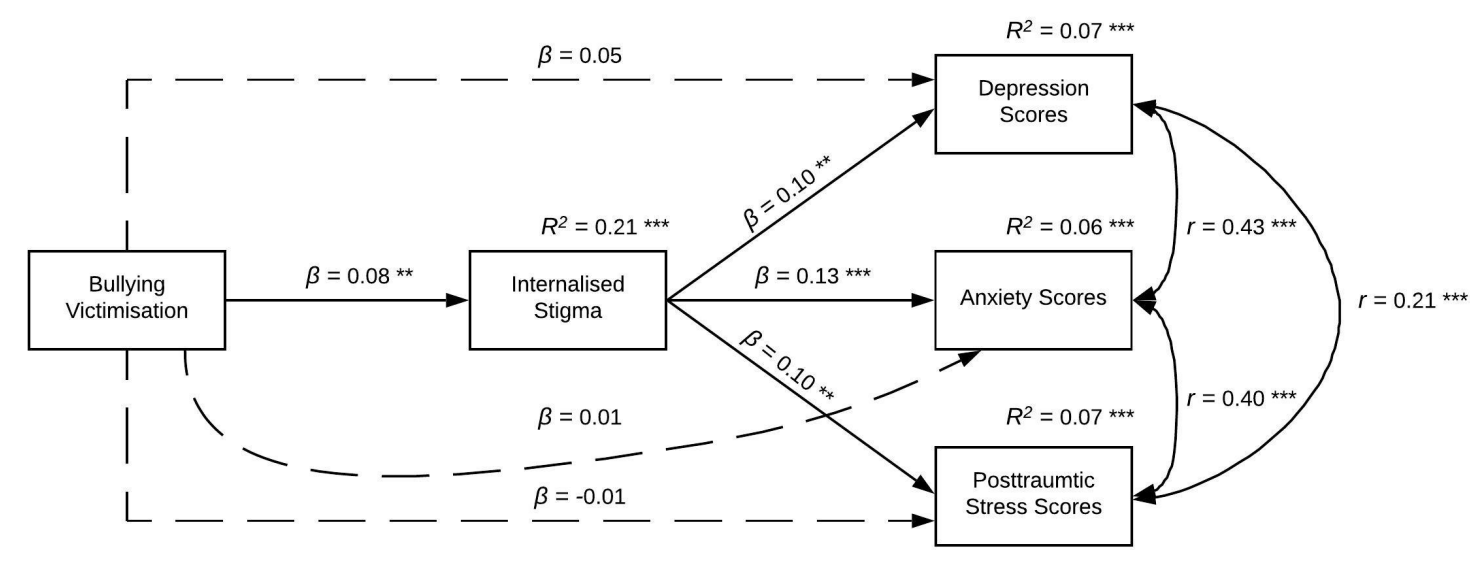


Bullying, stigma, and mental health in adolescents living with HIV 28

\section{Author Statement}

Contributors: As first author, MB took the lead in conceptualizing and writing the paper and conducted the statistical analysis. LC, ET, MC, MP, and EN discussed the results and provided conceptual feedback on the draft manuscript. LC and ET had key roles in designing the research study and managing the research data. LC, MB, ET, and MP contributed to the research design and to the choice of measurement tools. LC, ET, MC, and MP were involved in the management of the field research. All authors contributed to and have approved the final manuscript. None of the authors have any conflicts of interest to declare.

Role of the Funding Source: This research was funded by the Nuffield Foundation (under grant CPF/41513); UK Research and Innovation Global Challenges Research Fund Accelerating Achievement for Africa's Adolescents (Accelerate) Hub (Grant Ref: ES/S008101/1); the Evidence for HIV Prevention in Southern Africa (EHPSA), a UK aid programme managed by Mott MacDonald; Janssen Pharmaceutica N.V., part of the Janssen Pharmaceutical Companies of Johnson \& Johnson; International AIDS Society (CIPHER grant 155-Hod); the John Fell Oxford University Press Research Fund (103/757 and 161/033); European Research Council; the University of Oxford's ESRC Impact Acceleration Account [IAA-MT13-003; 1602-KEA-189; K1311-KEA-004];; Philip Leverhulme Trust (PLP-2014-095); the Regional Inter-Agency Task Team for Children Affected by AIDS Eastern and Southern Africa (RIATT-ESA) UNICEF Eastern and Southern Africa Regional Office (UNICEF-ESARO); Research England [0005218]; ); the National Research Foundation South Africa [RES-062-23-2068] and Oak Foundation [R46194/AA001]. Additional support for MB was provided by the National Health and Medical Research Council, Australia (Investigator Grant 1173043) and a New Independent Researcher 
Infrastructure Award from the Department of Health (Western Australia). Additional support for LC was provided by the European Research Council (ERC) under the European Union's Seventh Framework Programme [FP7/2007-2013]/ERC grant agreement no. 313421. EN was supported by a Curtin University Research Fellowship. ET received additional support from the International AIDS Society through the CIPHER grant [2018/625-TOS]. The views expressed in written materials or publications do not necessarily reflect the official policies of the International AIDS society. Research reported in this publication was supported by the Fogarty International Center, National Institute on Mental Health, National Institutes of Health under Award Number K43TW011434 (ET). The content is solely the responsibility of the authors and does not represent the official views of the National Institutes of Health. The funding sources did not have any involvement in the writing of this paper or in the decision to submit the article. The views expressed in this publication are those of the authors and do not necessarily reflect the official policies of the funders.

Acknowledgements: We are very grateful to the adolescents living with HIV and their caregivers who have generously shared their time and experiences for the purposes of this research. 